\title{
Medical errors: The performance gap in hypoplastic left heart syndrome and physiologic equivalents?
}

Frederic Jacques, MD, MSc, FRCSC, Vijay Anand, MD, Edward J. Hickey, MD, Yasuhiro Kotani, MD, PhD, Mrinal Yadava, MBBS, Abdullah Alghamdi, MSc, MD, Christopher A. Caldarone, MD, Andrew N. Redington, MD, Steven Schwartz, MD, and Glen S. Van Arsdell, MD

Background: The frequency and impact of medical errors during staged palliation are unknown.

Methods: All patients with hypoplastic left heart syndrome and physiologic equivalents $(\mathrm{N}=191)$ who underwent staged palliation (2001-2011) were studied. Stage 1, interstage, and stage 2 were reviewed to identify diagnostic, technical, judgment, and management errors. The impact of errors on transplant-free survival was examined by parametric competing risks and risk-adjusted regressions using bootstrapping.

Results: Stage $1(\mathrm{~N}=191)$ errors $(\mathrm{n}=111,58 \%)$ were common and predominantly intraoperative $(\mathrm{n}=84$, $44 \%)$ or postoperative $(n=43,23 \%)$. Postoperative errors were determinants of death/transplant (hazard ratio, $1.7 ; P=.01)$, whereas technical errors $(\mathrm{n}=65,34 \%)$ were not, but they delayed recovery and discharge (extra 24 days approximately, $P=.0024)$. Postoperative stage 1 errors led to decrements in total strategy success of approximately $30 \%(78 \%$ vs $48 \%, P=.004)$. Stage $2(\mathrm{~N}=134)$ errors $(\mathrm{n}=66,49 \%)$ were common. Intraoperative errors were the most prevalent $(\mathrm{n}=61,46 \%)$ but did not compromise survival. Postoperative errors $(\mathrm{n}=11,8 \%)$ were determinants of death/transplant (hazard ratio, 2.4; $P<.0001)$. Interstage errors $(\mathrm{n}=21$, $16 \%$ ) led to twice the intensive care unit stay (16 vs 7 days, $P<.0001)$ and hospital stay (30 vs 17 days, $P<.02)$ after stage 2 . Overall, a child presenting with ideal morphology and managed with no postoperative errors at stage 1 or 2 would have a predicted late survival in excess of $80 \%$.

Conclusions: Technical errors are common and delay recovery. Their effects on survival are mitigated. Intraoperative judgment errors are associated with strategy failure in a univariate model and lead to increased postoperative errors in a multivariate model. Postoperative errors are independently associated with a decrease in univentricular strategy survival. (J Thorac Cardiovasc Surg 2013;145:1465-75)

Hypoplastic left heart syndrome (HLHS) and physiologic equivalents are composed of various types of complex anatomy and physiology. The surgical options and medical treatment are similarly complex. Because medical complexity is a known association with medical error, ${ }^{1}$ it is conceivable that treatment errors occurring in patients with HLHS may lead to deleterious outcomes.

Up to one third of children undergoing univentricular palliation for HLHS will die or undergo cardiac transplantation before transitioning to a cavopulmonary connection. ${ }^{2}$ Efforts to understand strategy failures have focused on morphologic and functional risk factors. Such risks are now well understood but are generally immutable. Efforts have

From the Labatt Family Heart Centre, The Hospital for Sick Children, University of Toronto, Toronto, Ontario, Canada.

Disclosures: Authors have nothing to disclose with regard to commercial support.

Read at the 92nd Annual Meeting of The American Association for Thoracic Surgery, San Francisco, California, April 28-May 2, 2012.

Received for publication April 30, 2012; revisions received Nov 26, 2012; accepted for publication Dec 18, 2012; available ahead of print Feb 22, 2013.

Address for reprints: Glen S. Van Arsdell, MD, FRCSC, Division of Cardiovascular Surgery, Labatt Family Heart Center, The Hospital for Sick Children, Toronto, ON, Canada, M5G 1X8 (E-mail: glen.vanarsdell@sickkids.ca). $0022-5223 / \$ 36.00$

Copyright (c) 2013 by The American Association for Thoracic Surgery http://dx.doi.org/10.1016/j.jtcvs.2012.12.065 also focused on refining surgical techniques and preoperative and postoperative care. Levels of improvement to outcomes have been disappointing in the last decade..$^{2-4}$

The purpose of this study was to evaluate patient course for errors that might affect patient outcome. We hypothesized that human errors could translate into delayed recovery and increased risk of death or transplantation. Such errors represent a realistic opportunity for improving outcomes as they might be predicted, avoided, or mitigated. Study aims were to (1) review and identify preoperative, intraoperative, and postoperative errors (PEs) in diagnosis, judgment, clinical management, or surgical technique at both stages 1 and 2; (2) explore the impact of these medical errors on recovery and transplant-free survival; and (3) define patterns of errors among the surgical strategies used.

\section{MATERIALS AND METHODS Overview}

This investigation studied all 191 infants in whom staged surgical palliation for HLHS (or analogous physiology) was attempted between January 2001 and January 2011 at The Hospital for Sick Children, Toronto. Surgical strategies included the conventional Norwood operation (including both the modified Blalock-Taussig shunt and right ventricular to pulmonary artery conduit [Sano]) or hybrid approaches (with or without reverse Blalock-Taussig shunt) that have been selectively used for the last 7 years. Hybrid procedures performed to achieve hemodynamic 


\section{Abbreviations and Acronyms \\ HLHS $=$ hypoplastic left heart syndrome \\ ICU = intensive care unit \\ $\mathrm{PE} \quad=$ postoperative error}

stability while awaiting transplant were excluded. ${ }^{5}$ Each patient's course was summarized for the study time frame. Charts were reviewed by senior clinical fellows (F.J., V.A., Y.K.) who summarized individual patient hospitalization and outpatient courses into objective abstracts. The abstracts comprised complete anatomic details, imaging reports, decision-making process, operative or interventional details (including any revisions), postoperative course (including hemodynamic support, ventilatory support time, extubation or chest closure, and physiologic problems), transition to the ward, residual lesions, discharge planning (including medication), and outpatient follow-up. Electronic charts, including clinic letters, clinical conference decision notes, echocardiography, diagnostic imaging, catheterization and operative reports, perfusion records, intensive care and cardiology ward daily progress notes, all paramedical therapists' notes, laboratory values (including mixed venous oxygen saturations and lactate levels), and pathology reports, on every patient were reviewed until 10 months of age if on the staged surgical palliation pathway or until cardiac transplantation or death. Follow-up was complete in all patients. Abstracts were reviewed by a senior staff surgeon (G.S.V.) to identify and classify any errors in diagnosis, judgment, technical performance, and management. When required, questions from the reviewer were addressed by the abstractors and the case was reviewed again. Any finding that was less than optimal (anatomic or physiologic), that was not considered to be part of a "normal patient course" (or the primary presenting disease state), and that occurred as a result of a provider decision or intervention was labeled an error. Adverse events that did not result from a clinical decision or intervention were not labeled errors, for example, the development of renal dysfunction after an uncomplicated procedure would not be considered an error, whereas the need to reopen a chest because of premature chest closure-related hemodynamic compromise would. For the purpose of this study, we defined the optimal and normal course as follows: (1) preoperative: intent to or manage to have preoperative stability (ie, if necrotizing enterocolitis, renal failure, or sepsis occurs, an allowance for recovery before surgery). (2) intraoperative: anesthetic induction with hemodynamic stability and line insertion without complication, 1 period of cardiac ischemia, and cardiopulmonary bypass (including any intermittent circulatory arrest and regional cerebral perfusion) for the operation. Chest closure may or may not be performed. (3) postoperative: modest doses of inotropic and vasodilatory support; no issues of tamponade or major bleeding; if open, chest closure within 1 week and successful with 1 attempt; 1 successful extubation attempt; no cardiac arrest; no use of extracorporeal membrane oxygenation; no development of postoperative necrotizing enterocolitis or sepsis; medically manageable Qp:Qs; and transition to the ward and home without return and all occurring within a time frame of 4 to 5 weeks or less. An optimal anatomic condition was intraoperative and postoperative imaging demonstrating no arch obstruction, no shunt issues, no aortopulmonary connection flow acceleration, no pulmonary artery stenosis, and cardiac contractility that at least matched the preoperative state. For hybrid stage I, pulmonary artery stenosis was part of the planned procedure. A similar definition was used for stage II but with compressed timeframes and the addition of mild plus or less atrioventricular valve regurgitation. We defined an interstage optimal course to be no readmissions or interventions required and no need for cardiac rescue. The timing of the error with respect to time point in each patient's course was noted. The link and the sequence between errors, and the error impact on mortality and morbidity were assessed clinically by performing root cause analysis. The strength of causal link to each patient's course was estimated as follows: (1) no link to death, (2) possible link to death (ie, it is conceivably related but not likely), (3) mild relation to death (ie, it had an influence but little), and (4) direct relation to death. The impact of errors was explored in multivariate parametric competing risk models of transplant-free survival. The study was approved by The Hospital for Sick Children research ethics board, and consent requirement was waived.

\section{Statistical Methodology}

Survival analyses were performed via parametric time-related competing risks techniques using multiple hazard phases as previously described. ${ }^{6,7}$ All analyses were undertaken using SAS statistical software (SAS Institute Inc, Cary, NC) using parametric HAZARD macros (www. clevelandclinic.org/heartcenter/hazard). After stage 1, every child is at risk of transitioning to any of the mutually exclusive competing end states of death, transplantation, or successful transition to stage 2. Each of these end states can be modeled independently and subjected to riskhazard analysis. However, because transplantation represents failure of strategy, it was combined with death as "failures" of surgical palliative strategy.

\section{Risk-Hazard Analyses}

For risk-hazard analyses, variables were tested in a univariate fashion and subsequently multivariable (risk-adjusted) fashion using parametric automated forward stepwise regression. Variables were processed as previously described. ${ }^{7}$ All continuous variables were tested for transformations that improved model fit. Reliability of variables reaching statistical significance $(P<.05)$ was tested using bootstrap resampling in which automated random training datasets are generated $(\mathrm{N}=1000)$ against which the variables are tested. Inclusion of the variable in approximately $50 \%$ or more of training datasets is interpreted as an indicator of good reliability. ${ }^{8}$ Many variables that reach statistical significance in clinical regression analyses are poorly reliable in that they are not statistically significant when tested in other similar datasets. Bootstrap resampling tests the "generalizability" of a variable to the wider study population by describing both its significance and reliability.

Logistic and linear (forward stepwise) regression models were used to identify statistically significant determinants of binary and continuous variables, respectively (SAS statistical software), which were then bootstrap tested for reliability. For other descriptive statistics, comparisons or means or proportions were made using the Student $t$ test or Fisher exact test.

\section{RESULTS}

\section{Procedures and Outcomes}

The study cohort of 191 infants had aortic atresia $(\mathrm{N}=75 ; 40 \%)$, aortic stenosis $(\mathrm{N}=61 ; 32 \%)$, doubleinlet left ventricle/absent right atrioventricular connection associated with transposition of the great arteries $(\mathrm{N}=40 ; 21 \%)$, and unbalanced atrioventricular septal defect $(\mathrm{N}=15 ; 8 \%)$. All 191 infants underwent a stage 1 procedure $($ Norwood $=142,74 \%$; hybrid $=49,26 \%)$.

\section{Stage 1}

The Norwood operation was undertaken with a median myocardial ischemic time of 62 minutes (31-250 minutes) and the use of selective cerebral perfusion and intermittent circulatory arrest. Pulmonary blood flow was via a modified Blalock-Taussig shunt ( $\mathrm{N}=116,82 \%)$ or Sano modification $(\mathrm{N}=26,18 \%)$. Early in the decade, the Brawn all in situ technique ${ }^{9}$ was used for arch reconstruction in 7 
patients. Subsequently, the interdigitating technique was used. ${ }^{10}$ Intraoperative revisions were required in 42 patients $(30 \%)$, including shunt $(\mathrm{N}=24)$, pulmonary arteries $(\mathrm{N}=11)$, Damus-Kaye-Stansel anastomosis $(\mathrm{N}=11)$, and arch revision $(\mathrm{N}=9)$. Revisions were performed on the basis of direct clinical concern or findings on intraoperative echocardiography that were thought to be important to clinical outcome. Intraoperative implementation of extracorporeal membrane oxygenation support was necessary in 2 children. Hybrid stage 1 strategies were undertaken as previously described with the selective use of a "reverse Blalock-Taussig shunt" in 20 patients $(40 \%)$ to protect against retrograde arch malperfusion. ${ }^{11}$ Of all 191 stage 1 procedures, 34 patients $(18 \%)$ required extracorporeal membrane oxygenation support at some point in the postoperative period.

Outcomes after stage 1. Time-related outcomes after stage 1 are shown in Figure 1. In-hospital mortality at stage 1 was $16 \%(\mathrm{~N}=31 / 191)$. Before stage 2,11 other patients $(6 \%)$ died and 14 patients $(7 \%)$ were referred for transplantation. Successful transition to stage 2 occurred in 134 patients $(70 \%)$.

Errors surrounding stage 1 procedure. Errors surrounding stage 1 affected 111 of the 191 patients $(58 \%)$ and are summarized in Table 1 . Ten patients $(5 \%)$ had a preoperative error, 84 patients $(44 \%)$ had intraoperative error, and 43 patients had postoperative error $(23 \%)$. Intraoperative errors were related to clinical judgment or technical performance. The most common errors are presented in Table 2. The 3 most common intraoperative judgment errors were

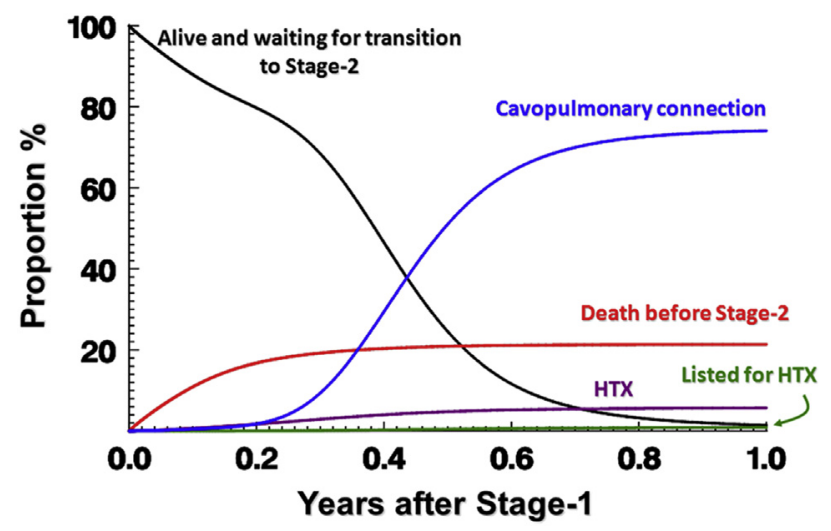

FIGURE 1. Competing outcomes after all 191 stage 1 interventions. Immediately after stage 1 , all $100 \%$ of infants are alive and have not yet transitioned to stage 2 or any other end state. Gradually with time, however, children transition at different rates to one of several mutually exclusive and competing end states: death, transplantation, or otherwise stage 2 . One year after stage $1,74 \% \pm 6 \%$ had successfully transitioned to stage $2,21 \% \pm 4 \%$ had died before stage $2,6 \% \pm 2 \%$ had undergone cardiac transplantation, and an additional $1 \%$ had been accepted on the waiting list for transplantation. Lines represent continuous point estimates of parametric models. HTX, Heart transplantation.
(1) decision to close the chest with subsequent hemodynamic compromise $(\mathrm{N}=10 / 191 ; 5 \%)$; (2) not recognizing or addressing shunt inflow concern not related to the anastomosis but to the particular anatomy of the patient (eg, a very large or unusually small innominate artery that could affect shunt flow) $(\mathrm{N}=9 / 191 ; 5 \%)$; and (3) selecting the wrong size for a shunt considering the body surface area of the patient $(\mathrm{N}=6 / 191 ; 3 \%)$. The 3 most common intraoperative technical errors were related to (1) systemicpulmonary shunt construction (ie, anastomotic problem) $(\mathrm{N}=21 / 191 ; 11 \%) ;(2)$ coronary artery malperfusion $(\mathrm{N}=7 / 191 ; 4 \%)$; and (3) obstructive aortic arch reconstruction $(\mathrm{N}=11 / 191 ; 6 \%)$. Most intraoperative errors were not detected at the time- $84 \%$ of judgment errors $(\mathrm{N}=21 / 25)$ and $63 \%$ of technical errors $(\mathrm{N}=41 / 65)$ went undetected. Of the technical errors, 27 were addressed after recognition in the postoperative period $(\mathrm{N}=27 / 65$; $42 \%)$. PEs occurred more commonly in the intensive care setting $(\mathrm{N}=22 / 191 ; 12 \%)$ than during ward care $(\mathrm{N}=4 /$ 191). The 3 most common errors in the intensive care unit (ICU) were (1) a delay in recognizing or managing a clinical scenario (eg, latent sepsis or low cardiac output syndrome) ( $\mathrm{N}=16 / 191 ; 8 \%) ;(2)$ a failed attempt of delayed sternal closure $(\mathrm{N}=11 / 191 ; 6 \%)$; and (3) errors related to airway devices and extubation $(\mathrm{N}=4 / 191 ; 2 \%)$. PEs were considered foreseeable in $77 \%$ of patients $(\mathrm{N}=33 / 43)$ after stage 1. Errors were defined as foreseeable when a patient's preceding condition and available information to the clinician might have predicted a less than optimal course. For example, an intubated patient presents with a moderate and not drained pleural effusion noted by the radiologist but not by the clinical team that includes surgeons, intensivists, and cardiologists (all according to the notes). The patient is extubated, fails extubation, is reintubated, has a chest tube inserted, and is reextubated within hours. That is, a foreseeable error as recognition of the effusion before extubation might have led to a different approach to the patient. The effusion per se was an indirect complication of the surgery but not an error. Of the 43 patients with a PE, 20 previously had an intraoperative error $(47 \%)$. In multivariate analysis, intraoperative judgment errors led to increased $\mathrm{PE}(\mathrm{PE}+0.87 ; P=.03)$.

Impact of errors on stage 1 outcomes. Table 3 presents univariate and multivariate risk factors for strategy failure after stage 1 . The occurrence of any type of error during stage 1 resulted in more than twice the risk of strategy failure with a high degree of significance and reliability $(P=.0009$; $76 \%$ ) in risk-adjusted models. Postoperative errors were the most important and remained strong, reliable, and independent determinants of strategy failure even after adjusting for patient complexity in multivariate models $(P=.01,47 \%)$. Children in whom PEs occurred exhibited decrements in transplant-free survival of approximately $15 \%$ (Figure 2 , A). In risk-adjusted models, foreseeable PEs are the most 
TABLE 1. Prevalence of errors occurring around the stage 1 procedure

\begin{tabular}{|c|c|c|c|c|c|}
\hline \multirow[b]{2}{*}{ Error } & \multicolumn{2}{|c|}{ Prevalence overall $(\mathrm{N}=191)$} & \multicolumn{2}{|c|}{ Prevalence $(\%)$} & \multirow[b]{2}{*}{$\boldsymbol{P}$} \\
\hline & $\mathbf{N}$ & $\%$ & Failure $(\mathbf{N}=\mathbf{5 6})$ & Success $(\mathbf{N}=135)$ & \\
\hline Any stage 1 error & 111 & 58 & 75 & 51 & .002 \\
\hline Preoperative stage 1 error & 10 & 5 & 7 & 4 & .48 \\
\hline Relating to pre-stage 1 intervention & 5 & 3 & 4 & 2 & .63 \\
\hline Relating to co-diagnosis & 2 & 1 & 0 & 2 & .36 \\
\hline Intraoperative stage 1 error & 84 & 44 & 57 & 39 & .025 \\
\hline Error in judgment & 25 & 13 & 23 & 9 & .017 \\
\hline Technical error & 65 & 34 & 38 & 33 & 62 \\
\hline Error in monitoring or assessment* & 3 & 2 & 4 & 1 & .21 \\
\hline Error relating to pulmonary arteries & 10 & 5 & 9 & 4 & .16 \\
\hline Error constructing systemic-pulmonary shunt & 21 & 11 & 13 & 10 & .80 \\
\hline Incorrect shunt size & 6 & 3 & 7 & 1 & .06 \\
\hline Arch stenosis or obstruction & 11 & 6 & 11 & 2 & .02 \\
\hline Error relating to coronary arteries & 7 & 4 & 4 & 4 & .96 \\
\hline Error relating to pulmonary veins & 2 & 1 & 2 & 1 & .50 \\
\hline Error relating to hemostasis & 4 & 2 & 0 & 3 & .33 \\
\hline Error relating to ductus & 4 & 2 & 0 & 3 & .33 \\
\hline Inappropriate sternal closure & 10 & 5 & 5 & 5 & .96 \\
\hline Ineffectual de-airing & 2 & 1 & 2 & 1 & .50 \\
\hline Postoperative stage 1 error & 43 & 23 & 34 & 18 & .022 \\
\hline Error in judgment & 14 & 7 & 13 & 5 & .12 \\
\hline Errors occurring on critical care & 22 & 12 & 16 & 9 & .22 \\
\hline Errors occurring on ward & 4 & 2 & 5 & 1 & .07 \\
\hline Errors relating to extubation & 4 & 2 & 2 & 2 & .85 \\
\hline Delay in response to clinical condition & 16 & 8 & 13 & 7 & .25 \\
\hline Inappropriate discharge from critical care & 3 & 2 & 0 & 2 & .56 \\
\hline Delay in readmission to critical care & 2 & 1 & 2 & 1 & .50 \\
\hline Premature attempt at sternal closure & 11 & 6 & 11 & 4 & .08 \\
\hline Error relating to lines & 3 & 2 & 0 & 2 & .56 \\
\hline Error relating to ECMO & 2 & 1 & 4 & $\mathbf{0}$ & .09 \\
\hline Error relating to anticoagulation & 2 & 1 & 2 & 1 & .50 \\
\hline
\end{tabular}

Overall rates of stage 1 errors and comparative error rates in children who failed the univentricular strategy (death or transplantation) versus those who successfully transitioned to stage 2. $P$ values represent chi-square comparisons of proportions. Bold indicates variables with a $P$ value of $<.1$ that were entered into the regression model. ECMO, Extracorporeal membrane oxygenation. *Anesthetic management errors were included in this category.

important determinants of stage 1 failure $(\mathrm{PE}+0.84$; $P=.0054)$. Less strong but possibly still important are subcategories of intraoperative errors. Intraoperative undetected judgment errors were a determinant of strategy failure on univariate analysis $(\mathrm{PE}+1.05 ; P=.0013)$, as were detected technical errors $(\mathrm{PE}+0.83 ; P=.0086)$. Viewed in total (multivariate analysis), intraoperative technical errors (the most prevalent type of stage 1 error) were not associated with increased risk of strategy failure, which suggests that important technical errors-resulting in highly hemodynamically significant lesions-were recognized and addressed at the operation (eg, aortopulmonary connectionrelated coronary malperfusion, major shunt flow issues, and significant arch obstruction).

Complexity and errors. Because PEs were strong and reliable determinants of poor outcome, we sought predictors of these PEs. Aortic atresia $(P=.009)$, presence of bilateral superior vena cavae $(P=.02)$, multiple cardiopulmonary bypass runs $(P=.04)$, and intraoperative judgment errors
$(P=.04)$ were all important predictors that PEs would occur, even in multivariate risk-adjusted models. Therefore, more complex morphologic substrates and errors in surgical judgment seem to contribute to a high likelihood of intensive care errors and elevated risk of strategy failure.

Clinical estimation of errors' importance. With regard to the clinical importance estimate of the errors at stage 1 , of the 111 errors, $74(67 \%)$ were judged to have conferred some level of impact on morbidity or mortality. Of these 74 recognized impacts, $24(32 \%)$ were linked to death and $56(75 \%)$ were linked to identifiable morbidity. In the 24 errors linked to death, the strength of the causal link was possible in $2(8 \%)$, a mild relationship to death was deemed to have occurred in $13(54 \%)$, and a direct relation occurred in $9(38 \%)$.

Impact of errors on speed of recovery. Although intraoperative technical errors did not increase the risk of strategy failure, their occurrence was an important determinant of stage 1 recovery to discharge from an ICU or a hospital. 
TABLE 2. Most common errors at various time points

\begin{tabular}{|c|c|c|}
\hline Most common errors & \multirow[b]{2}{*}{$\mathbf{N}=191$} & \\
\hline Stage 1 & & \\
\hline \multicolumn{3}{|l|}{ Intraoperative judgment errors } \\
\hline $\begin{array}{l}\text { 1. Decision to close the chest with early } \\
\text { hemodynamic compromise }\end{array}$ & 10 & $5 \%$ \\
\hline $\begin{array}{l}\text { 2. Not addressing shunt inflow concern related } \\
\text { to particular anatomy }\end{array}$ & 9 & $15 \%$ \\
\hline 3. Selecting wrong size shunt for body surface area & 6 & $3 \%$ \\
\hline \multicolumn{3}{|l|}{ Intraoperative technical errors } \\
\hline 1. Systemic-pulmonary shunt anastomotic problem & 21 & $11 \%$ \\
\hline 2. Coronary artery malperfusion & 7 & $4 \%$ \\
\hline 3. Obstructive aortic arch reconstruction & 11 & $6 \%$ \\
\hline \multicolumn{3}{|l|}{ Intensive care management errors } \\
\hline 1. Delay recognizing/managing a clinical scenario & 16 & $8 \%$ \\
\hline 2. Failed attempt of delayed sternal closure & 11 & $6 \%$ \\
\hline 3. Failed extubation & 4 & $2 \%$ \\
\hline Interstage at stage 2 & $\mathbf{N}=\mathbf{1 3 4}$ & \\
\hline \multicolumn{3}{|l|}{ Interstage } \\
\hline 1. Delay to act on a restrictive atrial septum & 18 & $13 \%$ \\
\hline 2. Delay in recognizing and acting on a complication & 8 & $6 \%$ \\
\hline \multicolumn{3}{|l|}{ Stage 2} \\
\hline \multicolumn{3}{|l|}{ Intraoperative judgment errors } \\
\hline $\begin{array}{l}\text { 1. Atrioventricular valve regurgitation left } \\
\text { unaddressed }\end{array}$ & 1 & $1 \%$ \\
\hline 2. Pulmonary arterioplasty requiring 3 revisions & 1 & $1 \%$ \\
\hline $\begin{array}{l}\text { 3. Tricuspid valve replacement after } 3 \text { failed } \\
\text { repair attempts }\end{array}$ & 1 & $1 \%$ \\
\hline \multicolumn{3}{|l|}{ Intraoperative technical errors } \\
\hline 1. Residual pulmonary artery stenosis & 41 & $31 \%$ \\
\hline 2. Need for atrioventricular valve repair revision & 5 & $4 \%$ \\
\hline \multicolumn{3}{|l|}{ Intensive care management errors } \\
\hline 1. Preventable cardiac arrest & 6 & $4 \%$ \\
\hline Associated with airway management issues & 1 & $1 \%$ \\
\hline Transport from operating room to ICU & 1 & $1 \%$ \\
\hline Improper chest tube management & 1 & $1 \%$ \\
\hline 2. Delay performing an intervention & 4 & $3 \%$ \\
\hline
\end{tabular}

$I C U$, Intensive care unit.

Intraoperative technical errors resulted in an average delay in hospital discharge of more than 3 weeks versus when technical errors did not occur $(P=.0024)$.

\section{Stage 2}

Stage $2(\mathrm{~N}=134)$ was undertaken at a mean age of 5.7 months and included bidirectional cavopulmonary connection $(\mathrm{N}=89,66 \%)$, hemi-Fontan $(\mathrm{N}=11,8 \%)$, and comprehensive hybrid stage $2(\mathrm{~N}=30,22 \%)$. In-hospital mortality at stage 2 was $4 \%(N=6 / 134)$. Subsequent to stage 2,14 children $(10 \%)$ died and 5 children $(4 \%)$ were referred for transplantation. At latest follow-up, $14 \%$ of children who underwent stage 2 had failed the univentricular strategy.

Interstage errors. Of the 134 children who successfully transitioned to stage $2,29(22 \%)$ were subject to
TABLE 3. Impact of stage 1 errors on risk of univentricular strategy failure

\begin{tabular}{|c|c|c|c|}
\hline Risk factor & $\begin{array}{c}\text { Parameter } \\
\text { estimate }\end{array}$ & $\boldsymbol{P}$ & $\%$ Reliability \\
\hline \multicolumn{4}{|l|}{ Univariate } \\
\hline Any stage 1 error & +1.06 & .0009 & - \\
\hline Any intraoperative stage 1 error & +0.63 & .022 & - \\
\hline Intraoperative error in judgment & +0.97 & .0022 & - \\
\hline $\begin{array}{l}\text { Total number of intraoperative } \\
\text { errors }\end{array}$ & +0.36 & .037 & - \\
\hline Any PE & +0.82 & .0042 & - \\
\hline Aortic atresia & 0.78 & .0044 & - \\
\hline Mitral atresia & 0.6 & .0128 & - \\
\hline $\begin{array}{l}\text { Double-inlet left ventricle } \\
\text { (protective) }\end{array}$ & -2.1 & .0035 & - \\
\hline $\begin{array}{l}\text { Longer cardiopulmonary bypass } \\
\text { duration (min) }\end{array}$ & 0.003 & .0041 & - \\
\hline Intraoperative technical errors & - & NS & - \\
\hline Preoperative errors & - & NS & - \\
\hline Hybrid vs conventional surgery & - & NS & - \\
\hline \multicolumn{4}{|l|}{ Multivariate } \\
\hline $\begin{array}{l}\text { Double-inlet left ventricle } \\
\text { (protective) }\end{array}$ & -2.1 & .0039 & 81 \\
\hline $\begin{array}{l}\text { Longer cardiopulmonary bypass } \\
\text { duration (min) }\end{array}$ & +0.003 & .0097 & 55 \\
\hline Any PE & +0.71 & .0124 & 47 \\
\hline
\end{tabular}

management errors during the interstage period (between stage 1 discharge and admission for stage 2). Most of these errors were a delay to act on a restrictive atrial septum $(\mathrm{N}=18 / 134 ; 13 \%)$ or a delay in recognizing and acting on a complication $(\mathrm{N}=8 / 134 ; 6 \%)$. All restrictive atrial septum errors were hybrid strategies.

Impact of interstage errors. Errors sustained during the interstage time frame did not seem to influence the risk of failure. Nevertheless, interstage errors significantly delayed recovery after stage 2 : ICU discharge was 16 days versus 7 days $(P<.0001)$, and hospital discharge was 30 days versus 17 days $(P<.02)$.

Stage 2 errors. At the time of stage 2, errors occurred in 66 of the 134 patients $(49 \%)$, a similar error prevalence to stage 1 (Table 4$)$. Three patients $(2 \%)$ presented with a preoperative error: unrecognized respiratory infection, performance of an early bidirectional cavopulmonary shunt in the presence of bilateral superior vena cavae, and known atrioventricular valve regurgitation that was not addressed. The majority of errors at the time of stage 2 also occurred intraoperatively $(\mathrm{N}=61 ; 46 \%)$; only 11 infants $(8 \%)$ sustained postoperative stage 2 errors. Most common errors are presented in Table 2. In the intraoperative period, judgment errors occurred in 3 patients $(2 \%)$, and technical errors occurred in 58 patients $(43 \%)$. Judgment errors were atrioventricular valve regurgitation left unaddressed, a pulmonary arterioplasty requiring 3 revisions, and a tricuspid 

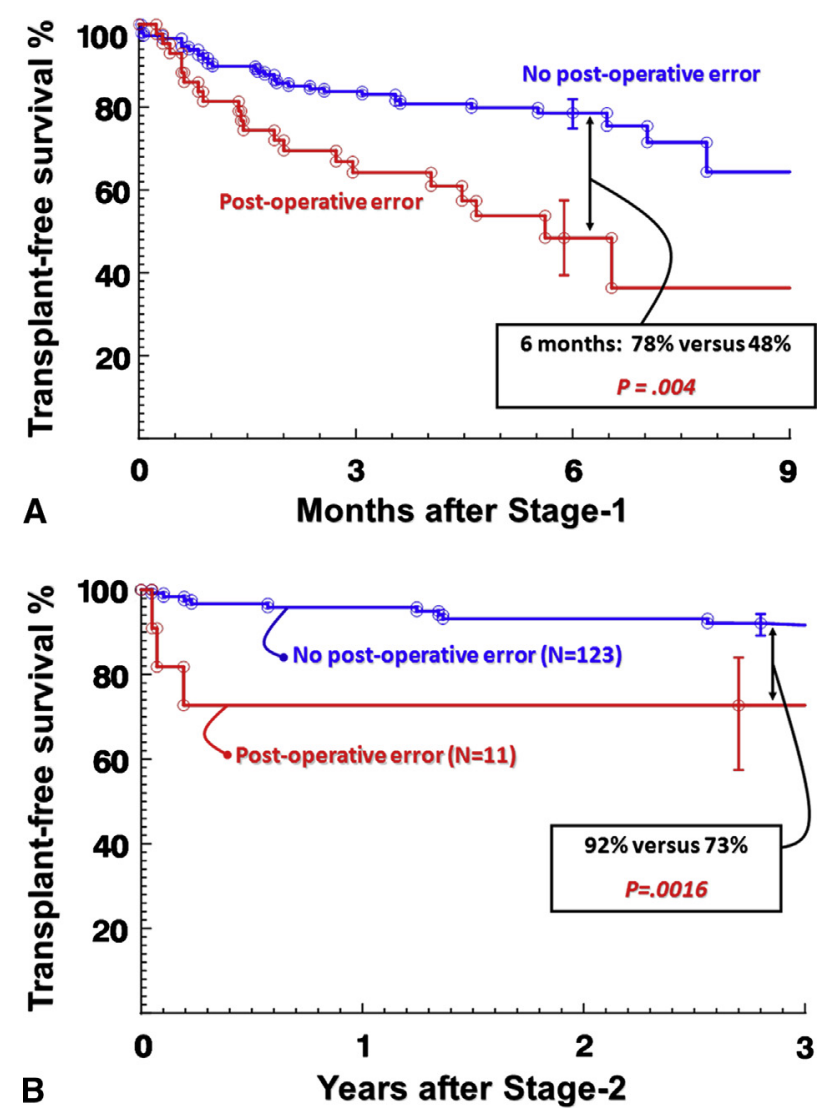

FIGURE 2. A, Impact of PEs on risk of univentricular strategy failure after stage 1. Actuarial freedom from interstage failure (death, cardiac transplantation, or listed for cardiac transplantation) after stage 1 in all 191 study patients, stratified by whether a PE occurred $(\mathrm{N}=43)$ or $\operatorname{did} \operatorname{not}(\mathrm{N}=148)$. The occurrence of a PE more than doubled the likelihood of univentricular strategy failure. B, Impact of PEs on risk of univentricular strategy failure after stage 2. Actuarial freedom from failure (death, cardiac transplantation, or listed for cardiac transplantation) after stage 2 in all 134 patients who underwent partial cavopulmonary connection, stratified by whether a PE occurred $(\mathrm{N}=11)$ or did not $(\mathrm{N}=123)$. The occurrence of a PE more than trebled the likelihood of univentricular strategy failure. Lines represent Kaplan-Meier estimates at the time of each event (circles).

valve replacement after 3 failed repair attempts. The most important technical errors were residual pulmonary artery stenosis $(\mathrm{N}=41 ; 31 \%)$ and the need for atrioventricular valve repair revision $(\mathrm{N}=5 ; 4 \%)$. Of the postoperative pulmonary artery stenosis issues, 20 occurred among the 100 Norwood procedures $(20 \%)$ and 21 occurred among the 34 hybrid procedures $(62 \%)$. In the postoperative period, 6 patients $(4 \%)$ had a cardiac arrest, which was related to airway management issues in $4(3 \%)$. Four other patients (3\%) had a delay in performing an intervention. Of the $11 \mathrm{PEs}, 6(4 \%)$ were determined to be related to judgment and $5(4 \%)$ were related to management strategy. Seven of the 11 patients had a preceding intraoperative error $(64 \%)$. Impact of errors on outcomes after stage 2. Risk factors for failure after stage 2 are shown in Table 5. Errors occurring at stage 1 did not have significant repercussions on failure after stage 2. Intraoperative errors at stage 2 also did not compromise outcomes. However, as was the case for stage 1 , PEs at stage 2 were important and reliable determinants of death or transplant $(\mathrm{PE}+2.9, P=.0016$, $76 \%$ reliability). Therefore, despite the low prevalence of postoperative stage 2 errors (only $8 \%$ ), their impact on strategy failure is marked. The occurrence of PEs at the time of stage 2 led to a decrease in late strategic success of approximately $20 \%$ (Figure 2, B). The small number of errors after stage 2 did not allow strong assertions regarding intraoperative error rescue and PE foresight.

Clinical estimation of errors' importance at stage 2. With regard to the estimate of clinical importance of the 66 errors at stage 2, $41(66 \%)$ were judged to have conferred some impact on morbidity or mortality. Four errors $(10 \%)$ were linked to death, and 38 errors ( $93 \%$ ) were linked to identifiable morbidity. The errors linked to death were thought to be related in 1 patient, mildly related in 2 patients, and directly related in 1 patient.

\section{Error Rates: Conventional Norwood Versus Hybrid}

Both Norwood and hybrid strategies were associated with a high rate of error at the time of stage 1, although this rate was significantly higher with Norwood surgery (65\% vs $40 \%$ respectively, $P=.001)$. This difference was especially marked for intraoperative errors $(P<.0001)$. However, this trend was reversed at stage 2 , where errors were then significantly more prevalent among hybrid approaches ( $74 \%$ vs $41 \%, P=.0014)$, particularly intraoperative technical errors $(P=.0012)$. Overall, neither early nor late freedom from strategy failure was different between hybrid and conventional Norwood approaches.

\section{Impact of Errors on Overall Strategy Failure}

Children who did not experience a PE at stage 1 or 2 exhibited a more than $15 \%$ higher rate of sustained strategic success (Figure 3) $(P=.04)$. A child without aortic atresia or bilateral superior vena cavae who is managed without any PEs at either stage has a predicted late ( $>3$ years) transplant-free survival of more than $80 \%$.

\section{DISCUSSION}

This study shows that the majority of children undergoing staged palliation for HLHS experience perioperative errors. Important determinants of adverse events, in all fields of medicine, are diseases that have high complexity and complex treatments. Although not all adverse events are caused by medical error, most have been identified as preventable. ${ }^{1,12,13}$ All surgical options for HLHS and physiologic equivalents represent complex surgical interventions for a complex anatomy and physiology. The resulting stage 1 balanced circulation leaves little room for tolerance of the high physiologic demands on the single ventricle. This 
TABLE 4. Prevalence of errors occurring around the stage 2 procedure $(\mathbf{N}=134)$

\begin{tabular}{|c|c|c|c|c|c|}
\hline \multirow[b]{2}{*}{ Error } & \multicolumn{2}{|c|}{ Prevalence overall $(N=134)$} & \multicolumn{2}{|c|}{ Prevalence $(\%)$} & \multirow[b]{2}{*}{$\boldsymbol{P}$} \\
\hline & $\mathbf{N}$ & $\%$ & Failure $(N=19)$ & Success $(N=115)$ & \\
\hline Any interstage error & 21 & 16 & 5 & 17 & .31 \\
\hline Error in clinical management & 18 & 13 & 5 & 15 & .46 \\
\hline Error in judgment & 2 & 1 & 0 & 2 & .56 \\
\hline Any stage 2 error & 66 & 49 & 63 & 47 & .22 \\
\hline Intraoperative stage 2 error & 61 & 46 & 58 & 43 & .32 \\
\hline Error in judgment & 3 & 2 & 5 & 2 & .37 \\
\hline Technical error & 58 & 43 & 53 & 41 & .46 \\
\hline Error relating to $\mathrm{AV}$ valve & 5 & 4 & 5 & 3 & .54 \\
\hline Error relating to coronary arteries & 1 & 1 & 0 & 1 & .68 \\
\hline Error relating to pulmonary arteries & 41 & 31 & 32 & 30 & .92 \\
\hline Error relating to SVC & 3 & 2 & 5 & 2 & .37 \\
\hline Sternal reentry error & 3 & 2 & 5 & 2 & .37 \\
\hline Phrenic nerve palsy & 2 & 2 & 0 & 2 & .56 \\
\hline Postoperative stage 2 error & 11 & 8 & 21 & 6 & .05 \\
\hline Error in judgment & 6 & 4 & 21 & 2 & .004 \\
\hline Error occurring on critical care & 5 & 4 & 0 & 4 & .36 \\
\hline Error occurring on ward & $\mathbf{0}$ & - & - & - & - \\
\hline
\end{tabular}

study was designed to identify, quantify, and qualify the impact of errors among these surgically palliated infants. Regardless of the surgical option chosen, hundreds of operative and intensive care maneuvers and decisions are required for each patient. Each decision or intervention is a point of patient risk. Because known risk factors for survival in surgically palliated patients with HLHS are often immutable morphologic or functional variables, ${ }^{2,3,14}$ we hypothesize that quantitative understanding of error data could identify target areas where improvement might

TABLE 5. Impact of errors on risk of strategy failure after stage 2

\begin{tabular}{lccc}
\hline \multicolumn{1}{c}{ Risk factor } & $\begin{array}{c}\text { Parameter } \\
\text { estimate }\end{array}$ & $\boldsymbol{P}$ & $\%$ Reliability \\
\hline Univariate & & & \\
$\quad$ Any postoperative stage 2 error & +1.5 & .0079 & - \\
$\quad$ PE in judgment & +2.44 & $<.0001$ & - \\
$\quad$ Double-inlet left ventricle & -1.99 & .054 & - \\
$\quad$ protective) & & & \\
Hemi-Fontan & +0.99 & .078 & - \\
Younger age at stage 2 & +0.01 & .0033 & - \\
$\quad$ Urgent or emergency stage 2 & +1.6 & .0011 & - \\
$\quad$ Errors occurring during stage 1 & - & $\mathrm{NS}$ & - \\
$\quad$ Errors occurring during interstage & - & $\mathrm{NS}$ & - \\
$\quad$ period & & & \\
$\quad$ Hybrid stage 2 vs conventional & - & $\mathrm{NS}$ & - \\
Multivariate & & & \\
$\quad$ Younger age at stage 2 & -.017 & .0019 & 77 \\
Any PE at stage 2 & +1.9 & .0016 & 76 \\
Hemi-Fontan & +1.32 & .0259 & 50 \\
\hline
\end{tabular}

Univariate and multivariate predictors of strategy failure (death or referral for cardiac transplantation) after stage $1(\mathrm{~N}=134)$. $N S$, Not significant; $P E$, postoperative error. be sought. Understanding and defining patterns for points of risk for error allow such a process while still recognizing that there can be "inevitability" about error that the present level of expertise and technology may not be able to mitigate.

\section{Preoperative Errors}

Preoperative errors were rare and had no discernible impact on survival.

\section{Intraoperative Errors}

Intraoperative errors were identified in approximately half of the patients. Univariate analysis demonstrated that

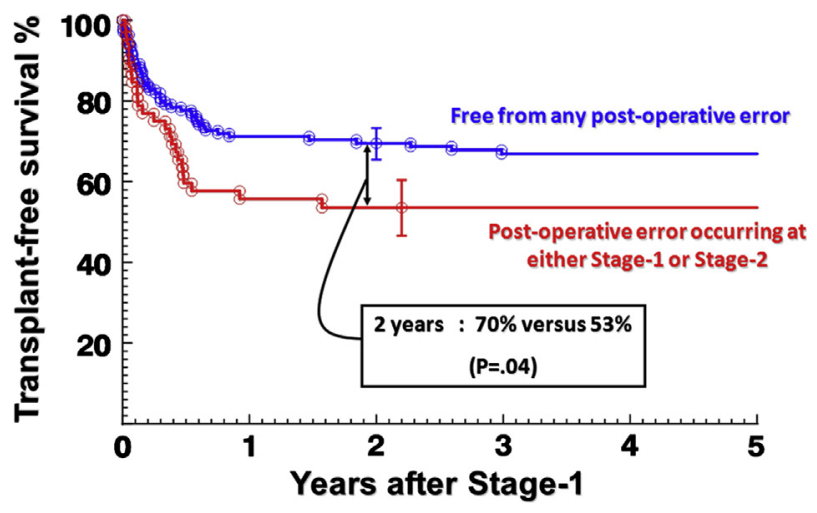

FIGURE 3. Benefit conferred by avoiding PEs at stage 1 and 2 procedures. Actuarial transplant-free survival for all 191 study patients, stratified by whether any type of PE occurred at any time $(\mathrm{N}=54)$ or did not $(\mathrm{N}=137)$. Lines represent Kaplan-Meier estimates at the time of each event (circles). 
intraoperative judgment errors led to a decrease in strategy success. In the multivariate model, they were linked to increased PEs. As a whole, intraoperative technical errors, although frequent, did not directly affect strategy survival. One potential reason is an institutional policy of assessing repair quality in the operating room using echocardiography. Aortic arch obstruction, coronary malperfusion, and aortopulmonary connection problems are immediately addressed. It was our intentional plan to eliminate lethal residual anatomic problems at the time of the original operative setting even if additional aortic crossclamp and cardiopulmonary bypass time was required. Less important anatomic problems may have only become evident in the postoperative timeframe. They were identified as residual lesions but flagged as an intraoperative error because they were considered the result of a less than optimal technical performance. These problems, such as mild pulmonary artery and shunt stenosis, might not be immediately noticed in the operating room because of the fidelity of imaging or the hemodynamic repercussions of general anesthesia, but still may have physiologic impact. ${ }^{15}$ Altogether, this underlines the importance of a good-quality repair as noted by other authors. ${ }^{16-18}$ Bacha and colleagues ${ }^{16}$ used predischarge echocardiography as a surrogate for intraoperative technical performance. They showed that residual lesions negatively affect patients' outcome. Bacha and colleagues' study and this study are not contradictory. For example, when examining patient-specific, known operative risks of longer and multiple bypass runs, incremental risk to strategy failure remains a consequence in a multivariate model, that is, technical performance still matters to survival. A less than optimal technical performance also makes postoperative care more difficult as manifest by the linkage to increased PE. Clearly, what transpires in the operating room is important to the level of illness and survival.

\section{Postoperative Errors}

PEs were the most important determinants of failure in this study. Patient-specific factors, such as aortic atresia or bilateral superior vena cavae influenced survival. After accounting for patients' risk status, PEs remained robust independent predictors of poor outcome. This fact is important, because it implies that postoperative management errors are not solely a function of patient complexity. However, patient complexity is also important given that the identified patient-specific risk factors also lead to a higher risk of error in critical care. This simply illustrates that more complex cases have a higher risk for error in management. ${ }^{1}$ A more specific concern regarding postoperative care is that it can be difficult to fully appreciate a patient's change in a fragile condition. This is highlighted by the fact that delays of recognizing and managing postoperative clinical scenarios constituted a large part of errors that occurred in the critical care unit. The recognition issues arise from the fidelity of diagnostic tools and the insidious pattern of presentation evolving over the postoperative recovery that can be counted in days and weeks. ${ }^{15-18}$

\section{Interlinkage Between Errors}

Results show that intraoperative errors increase intensive care and hospital length of stay. In other words, intraoperative errors lengthen the exposure time for PEs to occur. Reason ${ }^{19}$ and Cartney and colleagues ${ }^{20}$ term this "latent failure," which can be further illustrated by the conceivable scenario of both a judgment error and a technical error occurring in the operating room. Neither might be significant enough to be recognized at the time but could alter the ability of the patient to cope with later stress or management error. ${ }^{13,20}$ The latent effect can be seen clearly with interstage errors, where there was not a risk to failure of strategy, but there was a significant impact on the length of hospital stay during stage 2 .

\section{Importance of the Hinge Points}

Important areas of risk for error are transition points from one level of risk and monitoring to another, for example, chest closures, extubations, transfers out of the ICU, and hospital discharges. These "hinge points" are recognized sources of error in intensive care and may represent overall level of illness and complexity or even "flare up" of latent failures that occurred previously in the preoperative, intraoperative, and earlier postoperative periods. ${ }^{21-24}$ They also frequently involve decisions of expert judgment for patients who are physiologically marginal, for example, chest closure may occur in the ICU (a surgical and medical decision combined) but may fail and lead to worsening of the already marginal condition. Clearly defined guidelines and protocols for managing these transitions may improve the incidence of unfavorable decisions and interventions. At a minimum, protocols would allow for data-driven attempts at improving error incidence and allow for differentiation between a protocol error and a "normal course" adverse event. Hinge points represent an area for potential improvement. Further investigations should be devoted to better define these transitions in care and physiology, as well as to make these transitions better tolerated by these fragile patients.

\section{Hybrid Versus Norwood}

Some institutions reserve the use of the hybrid strategy for some defined anatomic variants, whereas at The Hospital for Sick Children hybrid stage 1 and Norwood stage 1 procedures are used for all the variants of HLHS. The pattern of error is different in the Norwood and hybrid procedures. For Norwood procedures, errors predominate during stage 1 . For hybrid procedures, errors are more prevalent during stage 2 . Overall survival for the 2 strategies was similar. Of note, an important difference between the 2 strategies is the prevalence of residual pulmonary artery 
stenosis in stage 2 conventional strategies $(20 \%)$ versus that in hybrid stage 2 strategies ( $62 \%)$. The long-term impact on the Fontan circulation remains to be determined.

\section{Study Limitations}

Medical errors are difficult to accurately identify. We attempted to use a thorough and introspective approach. Ironically, we could err in our estimate of error and may have overestimated its occurrence, although the literature would suggest that we would underestimate it. ${ }^{25}$ The indistinct difference between "errors" and some "complications" is also challenging. Leape and colleagues ${ }^{1}$ report that the line between preventable errors and ineluctable complications along the spectrum of adverse events is a moving target that evolves with increasing knowledge, that is, ineluctable complications of today may become known as medical errors tomorrow. In quality assessment and safety literature, it is recognized that complications are too frequently regarded as being immutable and underreported, especially those occurring during prolonged hospitalization. ${ }^{25}$ Self-fulfillment, a situation in which avoiding gaps in performance is not possible because of preceding events, is another limitation. Errors are interdependent and act synergistically on outcomes, and their intrinsic effect is sometime difficult to circumscribe.

Although the entire documented patient course was assessed through stage 2, transplantation, or death, some errors could have been missed because of a lack of documentation or granularity in documentation. This study is an initial attempt at understanding error in this cohort. Prospective evaluation will lead to enhanced understanding, particularly with respect to work organization such as leadership, hierarchy, and communication. A third-party concurrent observation would be of particular value.

\section{CONCLUSIONS}

In this study, the majority of children with HLHS and physiologic equivalents who underwent surgical palliation had an occurrence of medical error. Errors were most common in the intraoperative or postoperative windows. Technical intraoperative errors delayed patient recovery but did not influence the risk of strategy failure (likely because lethal errors were corrected in the same operative setting). Intraoperative judgment errors affected survival in a univariate analysis and led to increased PEs in a multivariate model. PEs were reliable determinants of strategy failure in a multivariable model. The occurrence of a PE decreases transplant-free survival by $15 \%$ to $20 \%$. Understanding patterns of errors and using strategies to decrease errors hold the possibility of improving patient outcome.

\section{References}

1. Leape LL, Brennan TA, Laird N, et al. The nature of adverse events in hospitalized patients: results of the Harvard medical practice study II. $N$ Engl J Med. $1991 ; 324: 377-84$
2. Feinstein JA, Benson DW, Dubin AM, et al. Hypoplastic left heart syndrome: current considerations and expectations. J Am Coll Cardiol. 2012;59(1 Suppl): S1-42. Review.

3. Ohye RG, Sleeper LA, Mahony L, et al. Comparison of shunt types in the Norwood procedure for single-ventricle lesions. N Engl J Med. 2010;362:1980-92.

4. Hickey EJ, Caldarone CA, McCrindle BW. Left ventricular hypoplasia: a spectrum of disease involving the left ventricular outflow tract, aortic valve, and aorta. J Am Coll Cardiol. 2012;59(1 Suppl):S43-54. Review.

5. Honjo O, Benson LN, Mewhort HE, et al. Clinical outcomes, program evolution, and pulmonary artery growth in single ventricle palliation using hybrid and Norwood palliative strategies. Ann Thorac Surg. 2010;87:1885-93.

6. Blackstone EH, Naftel DC, Turner MEJ. The decomposition of time- varying hazard into phases, each incorporating a separate stream of concomitant information. J Am Stat Assoc. 1986;81:615-24.

7. Hickey EJ, Nosikova Y, Zhang H, et al. Very low-birth-weight infants with congenital cardiac lesions: Is there merit in delaying intervention to permit growth and maturation? J Thorac Cardiovasc Surg. 2012;143:126-36.

8. Breiman L. Bagging predictors. Mach Learn. 1996;24:123-40.

9. Bu'Lock FA, Stümper O, Jagtap R, et al. Surgery for infants with a hypoplastic systemic ventricle and severe outflow obstruction: early results with a modified Norwood procedure. Br Heart J. 1995;73:456-61.

10. Burkhart HM, Ashburn DA, Konstantinov IE, et al. Interdigitating arch reconstruction eliminates recurrent coarctation after the Norwood procedure. J Thorac Cardiovasc Surg. 2005;130:61-5. Erratum in: J Thorac Cardiovasc Surg. 2005;130:1195. De Oliviera, Nilto C [corrected to De Oliveira, Nilto C].

11. Caldarone CA, Benson LN, Holtby H, Van Arsdell GS. Main pulmonary artery to innominate shunt during hybrid palliation of hypoplastic left heart syndrome. J Thorac Cardiovasc Surg. 2005;130:e1-2.

12. Leape LL, Lawthers AG, Breannan TA, Johnson WG. Preventing medical injury. Qual Rev Bull. 1993;19:144-9.

13. de Leval MR, Cartney J, Wright DJ, Farewell VT, Reason JT. Human factors and cardiac surgery: a multicenter study. J Thorac Cardiovasc Surg. 2000;119:661-72.

14. De Oliviera NC, Ashburn DA, Khalid F, et al. Prevention of early sudden circulatory collapse after the Norwood operation. Circulation. 2004;110(Suppl II):II-133-8.

15. Nathan M, Karamichalis JM, Liu H, et al. Intraoperative adverse events can be compensated by technical performance in neonates and infants after cardiac surgery: a prospective study. J Thorac Cardiovasc Surg. 2011;142:1098-107.

16. Bacha EA, Larrazabal LA, Pigula F, et al. Measurement of technical performance in surgery for congenital heart disease: the stage I Norwood procedure. J Thorac Cardiovasc Surg. 2008;136:993-7.

17. Karamichalis JM, del Nido PJ, Thiagarajan RR, et al. Early postoperative severity if illness predicts outcomes after stage 1 Norwood procedure. Ann Thorac Surg. 2011;92:660-5.

18. Karamichalis JM, Thiagarajan RR, Liu H, Mamic P, Gauvreau K, Bacha EA Stage I Norwood: Optimal technical performance improves outcomes irrespective of preoperative physiologic status or case complexity. J Thorac Cardiovasc Surg. 2010;139:962-8.

19. Reason JT. Human Error. Cambridge, UK: Cambridge University Press; 1990.

20. Cartney J, de Leval MR, Reason JT. The human factor in cardiac surgery: errors and near misses in a high technology medical domain. Ann Thorac Surg. 2001; 72:300-5.

21. Thomas AN, Panchagnula U, Taylor RJ. Review of patient safety incidents submitted from critical care units in England and Wales to the UK national patient safety agency. Anesthesia. 2009;64:1178-85.

22. Beckmann U, Bohringer C, Carless R, et al. Evaluation of two methods for quality improvement in intensive care: facilitated incident monitoring and retrospective medical chart review. Crit Care Med. 2003;31:1006-11.

23. Mahajan RP. Critical incident reporting and learning. Br J Anesth. 2010;10:69-75

24. Barach P, Johnson JK, Ahmad A, et al. A prospective observational study of human factors, adverse events, and patients outcomes in surgery for pediatric cardiac disease. J Thorac Cardiovasc Surg. 2008;136:1422-8.

25. Evans SM, Berry JG, Smith BJ, et al. Attitudes and barriers to incident reporting: a collaborative hospital study. Qual Saf Health Care. 2006;15:39-43.

\section{Discussion}

Dr Emile Bacha (New York, $N Y$ ). I have to compliment you and Dr Van Arsdell on a courageous study. It is never easy to admit having made a mistake, especially if a patient's life is on the 
line. However, it is also the first step toward learning from our mistakes, and you could call it a first step toward redemption. In fact, past errors properly documented can be seen as a form of negative knowledge.

Studies in the field of action science such as this one are difficult because they are inherently subjective. This leads me to my first question. How were the errors adjudicated? Was it by expert consensus or a single physician?

Dr Jacques. That is correct. For a contextual explanation, the multidisciplinary senior authors had previously performed a consensus-based review for 10 years of our institutional congenital cardiac surgery-related mortality. Through that process we had achieved a standardized approach for identifying and labeling error and thus were comfortable with the single senior review. This study was a subsequent study that looked not only at mortality (numerator) but also at the survivors (denominator) so that there could be a better understanding of the relation between all errors identified and their potential impact on outcomes.

Dr Bacha. So it was a single person, not a group of experts, deciding what was an error and what was not, correct?

Dr Jacques. Correct, but based on the methodology, that was including critical care and cardiology staff.

Dr Bacha. That is certainly one weakness of the study. How do you explain the seemingly contradictory information of the high stage 1 intraoperative error rate of $58 \%$, a high percentage of which was not detected; by your own article, $84 \%$ of judgment errors were not detected in the operating room, and $63 \%$ of technical errors were not detected, yet you have an acceptable operative mortality of $16 \%$ in the stage 1 operation. That is equivalent to other large centers. How do you explain that contradiction?

Dr Jacques. Every patient has an intraoperative echocardiographic evaluation in the operating room so that we can identify any major anatomic problem, such as Damus-Kaye-Stansel obstruction. We try to identify any occurrence of and solve all potentially lethal anatomic problems before leaving the operating room. I forgot the subsequent part of your question.

Dr Bacha. You have a high error rate and some recovery, but your outcomes are good. So you are saying your recovery, that is, your compensation mechanisms, are robust. That's what you're telling us, right?

Dr Jacques. Yes. It is important to understand that a number of minor technical issues, such as a small amount of pulmonary artery narrowing, were identified only on postoperative studies. Because they were less than optimal findings, they were labeled as a technical issue but stratified and estimated in the article as having no impact on clinical outcome.

Dr Bacha. Did you stratify errors by surgeon or level of experience?

Dr Jacques. It is a subanalysis that we are planning to do, but not at this point.

Dr Bacha. Your errors, again, were lumped into 2 fairly wide categories, technical or judgment for intraoperative errors and judgment and management for PEs. Of course, there are many types of errors. There are even specific taxonomy systems that allow you to place errors into specific categories, errors of omission, observational errors, communication errors, conceptual errors, and so forth. Would you consider reclassifying your errors in a more granular way to be able to hone in on specific problems better?
Dr Jacques. We used that classification to help us understand where we might look to make improvements. It is an interesting suggestion to look for more granular data; however, it is important to understand that some errors, such as communication errors, might be difficult to identify through a chart review.

Dr Craig Smith (New York, NY). If I worked in your ICU, I think I would be tempted to ask whether this is an exercise in blame-shifting, and I think there is an issue here that I may not be smart enough to understand statistically. You are describing 4 sequential events, and the fact that they are sequential might not influence the frequency of events in each episode, but it does seem to me possible that it would push the impact downstream and make anything that happens in the fourth episode appear more important than the things that happened previously. You touch on that possibility in demonstrating the high interrelatedness between factors, but I wonder if multivariable analysis is an adequate method for sorting out the sequential dependency of early events on subsequent events. Did you go through this with your statistician?

Dr Jacques. First, it is important to note that this study is not meant to assign blame. We didn't know this was going to be the finding of our study. Outcomes have been fairly steady for 10 or more years in this vulnerable physiology. We wanted to see if there were areas where we, as a team, could improve performance and thereby potentially enhance outcomes. In our estimate, the best way to approach this was to look broadly, with respect to time, areas of care, and definition of optimal. We are sensitive to the concept that this can be viewed as finger pointing. We would note that the surgeons are actively involved with the decision-making and interventions during the postoperative intensive care phase-it is a team effort. Further, a large number of the postoperative identified errors had to do with decision-making around chest closure (a surgical decision). The statistical analysis included preoperative/intraoperative information and PEs and was designed to identify independent factors associated with poor outcome. It could be that we are solving most of the life-threatening errors in the operating room by actively addressing echocardiogram-identified problems at the time. These patients are then rescued but have a propensity for more postoperative management challenges because they arrive in a less healthy state of physiology.

Dr Thoralf Sundt (Boston, Mass). I have a concern about your definition of error and the possibility that you have essentially contaminated it with the outcome. This raises potential for confusion between the occurrence of an error and the occurrence of an adverse outcome. For example, in an early slide you said you defined it as an error if the patient was over-anticoagulated and bled. But isn't it an error if the patient is over-anticoagulated regardless of whether he/she bleed, whether there is an adverse event or not? The importance of this distinction is that if you connect the 2 , if you require adverse event in the definition of error, then your result is a fait accompli; you are destined to show a relationship between errors and outcomes, which is the principal finding of your study.

Dr Jacques. This is an important point. We looked for adverse events that occurred outside of the usual course of the treated disease. We then determined if these had been affected by a clinical decision or intervention and if the adverse event was controllable or uncontrollable. If the event was not controllable, it was considered a natural complication and not labeled an error. Those that 
were controllable were considered an error. This is in line with the seminal publication of Leape and colleagues 20 years ago.

Dr Paul Kirshbom (Atlanta, $\mathrm{Ga}$ ). I was wondering with this very nongranular presentation if more granular analysis of your data presented you with any opportunities to take action. Did you identify a commonality in your ICU errors, for example, drug delivery in a large majority that could be affected by a change in your drug-delivery system or any other commonality you could change in the future?

Dr Jacques. Yes, we have identified areas to target. That was the ultimate goal, but I haven't been able to detail that aspect in the presentation. In the postoperative period, "hinge points" are important. We know that in the intensive care and anesthesia literature, all the transition points, such as transferring, transporting, and extubating the patient, are important periods. In our study, "hinge points of importance" are timing of delayed chest closure, timing of extubation, transfer to the ward, and the period surrounding the discharge of the patient. These are the most important areas where we will have to focus. We are now working on establishing guidelines to improve the care at these points.

\title{
COMMENTARY
}

\section{"Errare humanum est, perseverare autem diabolicum" -Lucius Annaeus Seneca, 4 BC to 45 AD}

\author{
Marc R. de Leval, MD, FRCS
}

Medical outcomes result from complex interactions among 3 sets of variables: those related to the diseases, those related to the treatments, and those related to the care providers. The majority of outcome researchers have concentrated on illness-specific or procedural-related variables. By and large, human factors related to the performance of care providers have not been included in those analyses. Yet investment in human factors has been shown to improve safety and enhance reliability in hightechnology industries in general and aviation in particular.

High-technology medicine such as cardiac surgery shares many properties with what is often referred to as complex social technical systems in which performance depends on complex individual technical and organizational factors and their interactions. ${ }^{1}$

In this issue of the Journal, Jacques and colleagues ${ }^{2}$ investigate the impact of human errors on team performance and outcomes of staged palliation of hypoplastic left heart syndromes and physiologic equivalents. Repairs of hypoplastic left heart syndromes are models of high-

\footnotetext{
From the Harley Street Clinic, Congenital Heart Centre, London, United Kingdom. Disclosures: Author has nothing to disclose with regard to commercial support.

Received for publication Feb 7, 2013; accepted for publication March 14, 2013; available ahead of print April 15, 2013

Address for reprints: Marc R. de Leval, MD, FRCS, the Harley Street Clinic, Congenital Heart Centre, 84 Harley St, 2nd Floor, London W1G 7HW, United Kingdom (E-mail: marc.deleval@hcahealthcare.co.uk).

J Thorac Cardiovasc Surg 2013;145:1475-6

$0022-5223 / \$ 36.00$

Copyright (C) 2013 by The American Association for Thoracic Surgery

http://dx.doi.org/10.1016/j.jtcvs.2013.03.010
}

technology surgery par excellence. They have a low error tolerance requiring a sophisticated organizational structure, the coordinated efforts of multiple individuals working in teams, and high levels of cognitive and technical performance.

The authors review the course summaries of 191 patients undergoing operations in a single institution over a 10 -year period. Technical and judgment errors at each stage of the Norwood strategy were extracted from these summaries. Human errors affected approximately $50 \%$ of the patients at both stage I and II of the Norwood strategy. At stage I, most intraoperative judgment errors are not detected at the time of the operation. Serious technical errors are recognized and addressed intraoperatively (30\% of patients at stage I underwent a revision of the repair). Those revisions were not associated with an increased risk of strategy failure (death or transplantation), but they delayed the postoperative recovery. Judgment errors led to an increase in postoperative errors.

Postoperative errors are the strongest and most reliable determinants of poor outcomes. The predictors of postoperative errors are the complexity of the morphologic substrate (more complex patients have a higher risk for error in their management), and errors in intraoperative surgical judgment increase the risk of postoperative errors. The majority of postoperative errors after stage I are foreseeable.

Intraoperative errors at stage II did not compromise outcomes, but despite their low incidence the postoperative errors did have an impact on outcomes.

This study conveys important messages. In the current state of the art, the treatment of complex congenital cardiac 